\title{
Space-time phononic crystals with anomalous topological edge states
}

\author{
Mourad Oudich,,${ }^{1,2, *}$ Yuanchen Deng, ${ }^{1}$ Molei Tao, ${ }^{3}$ and Yun Jing ${ }^{1, \dagger}$ \\ ${ }^{1}$ Department of Mechanical and Aerospace Engineering, North Carolina State University, Raleigh, North Carolina 27695, USA \\ ${ }^{2}$ Université de Lorraine, CNRS, Institut Jean Lamour, F-54000 Nancy, France \\ ${ }^{3}$ School of Mathematics, Georgia Institute of Technology, Atlanta, Georgia 30332, USA
}

(Received 14 July 2019; published 1 November 2019)

\begin{abstract}
It is well known that an interface created by two topologically distinct structures could host nontrivial edge states that are immune to defects. In this paper, we introduce a one-dimensional space-time phononic crystal and study the associated anomalous topological edge states when the phononic crystal's density is modulated both in space and time. While preserving the key topological feature of the system, the time modulation also duplicates the edge state mode across the spectrum, both inside and outside the band gap. It is shown that, in contrast to conventional topological edge states which are excited by frequencies in the Bragg regime, the time-modulation-induced frequency conversion can be leveraged to access topological edge states at a deep subwavelength scale where the entire phononic crystal size can be as small as $1 / 5.1$ of the wavelength. This feature could open another route for designing miniature devices that are based on topological physics.
\end{abstract}

DOI: 10.1103/PhysRevResearch.1.033069

\section{INTRODUCTION}

There has been a growing interest in topological phases since the discovery of topological insulators in quantum physics. One of the most intriguing phenomena associated with topological phases is the topological edge state, which is symmetry protected and is immune to local material imperfections. Topological phases have also recently been identified in classical systems such as in photonics [1-11], acoustics [12-28], and mechanics [29-33]. These topological phases harbor nontrivial wave modes that are stable against local perturbations, offering an intriguing solution for realizing robust, backscattering-immune wave propagation [12-23], as well as energy localization [24-28]. While most recent studies pertaining to topological edge states have focused on twodimensional (2D) [12-20,27-33] or even three-dimensional (3D) systems [21-23], one-dimensional (1D) systems [24-26] could still serve as a simple yet versatile platform for advancing our fundamental knowledge on topological physics. For the 1D case, the topological phase of the bulk band is known as the Zak phase [34,35]. Considering a design of a periodic structure with a band gap (BG), it is discovered that the continuous deformation of the structure with constant symmetry can produce closing and opening of the BG supported by a topological phase transition. This phase transition can be leveraged to design topologically protected edge states that reside between the initial structure and a deformed one.

\footnotetext{
*moudich@ncsu.edu

†yjing2@ncsu.edu
}

Published by the American Physical Society under the terms of the Creative Commons Attribution 4.0 International license. Further distribution of this work must maintain attribution to the author(s) and the published article's title, journal citation, and DOI.
Xiao et al. [24] demonstrated this mechanism in a 1D acoustic system where a topological edge state was created between two phononic crystals (PCs). Meanwhile, topological edge states are dictated by the geometrical symmetry of the system and occur at the topological BG where the wavelength is comparable with the structure periodicity [17]. This limits their functionalities to the Bragg scattering regime. Consequently, the resulting structure can be very bulky at low frequencies. This paper introduces another strategy to break down this barrier by imposing both space and time modulation (STM) on the medium properties. The STM could enable the engineering of topological phases in the time domain which provides an extra degree of freedom and therefore richer physics. There are two major findings in this study: (1) the emergence of anomalous topological edge states in the bulk band; and (2) the possibility to excite the edge state in the Bragg regime using ultralow frequencies whose wavelengths could be orders of magnitude greater than the periodicity of the PC. These features could bring about different designs of miniature devices based on topological phases for unconventional wave manipulation, excitation, and detection. Although the current study focuses on 1D acoustic systems, the theory developed here can readily be extended to higher dimensions and other wave-based systems.

STM of the medium properties has been proposed as a robust way to break reciprocity which is intrinsic in conventional dynamical systems. Consequently, nonidentical wave transmission in opposite directions has been achieved. Spacetime (ST) photonic crystals were in fact introduced decades ago [36-38] and the subject on nonreciprocity has recently been reinvigorated in the field of photonics for the control of electromagnetic waves using time variation of permittivity and/or permeability [39-43]. There have also been numerous works regarding STM in acoustics $[44,46]$ and elastic waves where the mechanical properties of the medium are ST modulated to achieve nonreciprocal wave propagation [47-50]. 


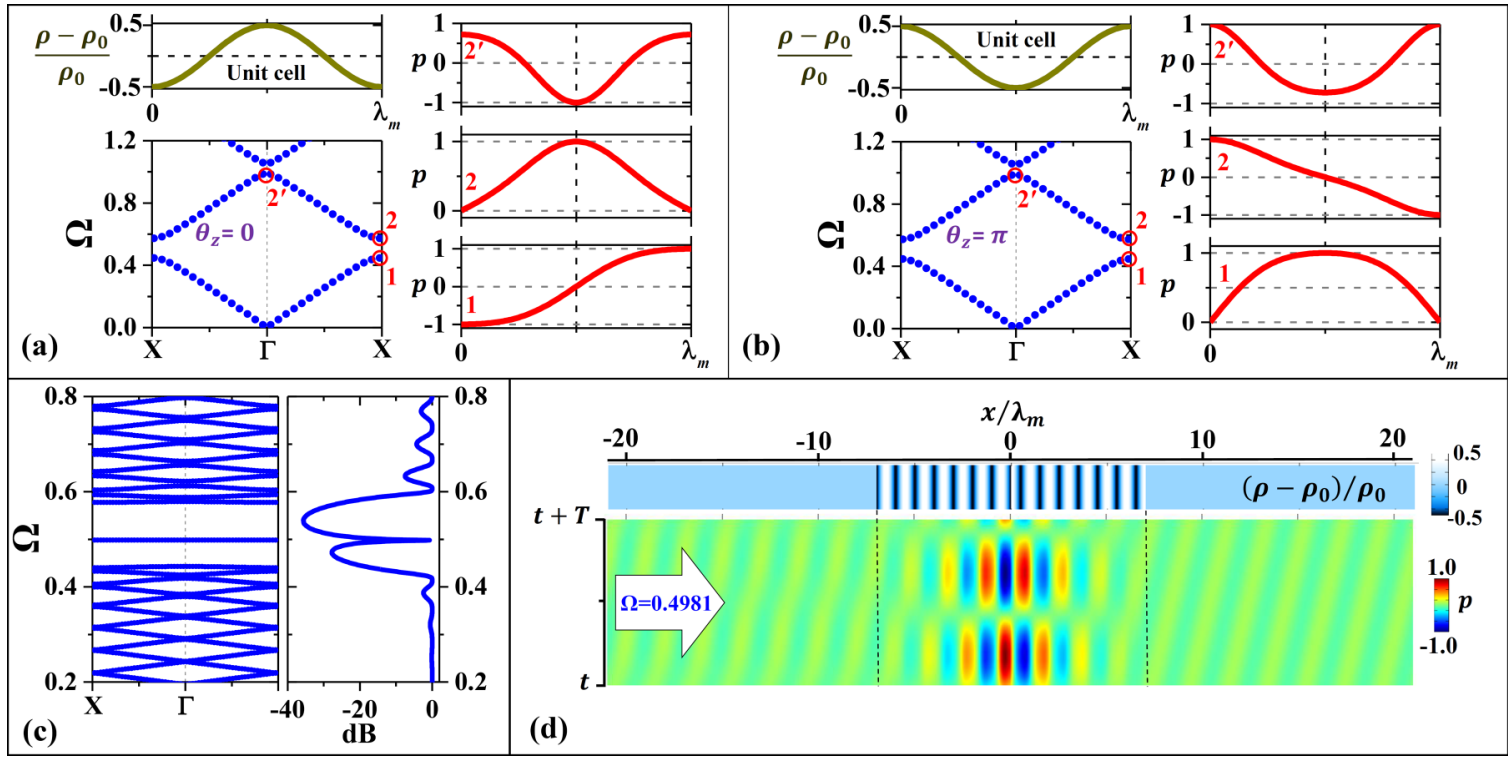

FIG. 1. (a) A stationary PC with density modulation of $\rho=\rho_{0}\left[1-\alpha \cos \left(k_{m} x\right)\right]$ along the unit cell $\left[0, \lambda_{m}\right]$ and its band structure (bottom left panel). Pressure fields are also plotted for the eigenmodes indicated by 1,2 , and $2^{\prime}$ in the band structure (right panels). (b) Same as (a) but for the second stationary PC with density modulation of $\rho=\rho_{0}\left[1+\alpha \cos \left(k_{m} x\right)\right]$. (c) Band structure for the system constructed by the two PCs where each PC consists of 7 unit cells: $\rho=\rho_{0}\left[1-\alpha \cos \left(k_{m} x\right)\right]$ for $x<0$ and $\rho=\rho_{0}\left[1+\alpha \cos \left(k_{m} x\right)\right]$ for $x>0$. The corresponding transmission spectrum is shown on the right, where an edge state with a high transmission can be observed at $\Omega=0.4981$. (d) Transmission configuration along the system (top panel) and the edge state pressure field for a single frequency excitation plotted against the time (bottom panel). In both panels, the vertical axis is the time and the horizontal axis is the space.

Meanwhile, it has been shown that external time-dependent perturbations can be used to achieve topological spectra in initially trivial systems at the equilibrium [6,16,51,52]. This class of systems is known to be the basis of Floquet topological insulators. A time-periodic potential can be also introduced to a Hermitian system which leads to nontrivial duplicated edge states in the band structure $[53,54]$.

In this work, in contrast to the approach for building Floquet topological insulators, we start from a stationary 1D system possessing nontrivial topological phases as well as topologically protected edge states inside a BG (Sec. II). This initial system is constructed from two space-modulated PCs exhibiting distinct Zak phases, i.e., 0 and $\pi$. We then introduce the time modulation into both PCs in a way that the Zak phase difference between the two PCs remains unchanged throughout the entire duration of time modulation [53]. The resulting PC is referred to as the space-time PC: a "living" PC that evolves in time. We consider two kinds of time modulations: space decoupled and space coupled. While the latter breaks reciprocity, the former conserves reciprocity (Secs. III and IV, respectively). The fundamental differences between the two modulations will be delineated. As will be shown below, frequency conversion occurs in the space-time PC, which gives rise to the duplication of edge states across the entire temporal spectrum. This is the underlying mechanism for the anomalous edge state discovered in this study.

\section{TOPOLOGICAL ONE-DIMENSIONAL PHONONIC CRYSTAL}

We first consider two PCs having a sinusoidal space modulation of the medium density $\rho(x)$. The governing equations in acoustics in 1D read [55]

$$
\frac{\partial p}{\partial x}=-\rho \frac{\partial v}{\partial t}, \quad \frac{\partial v}{\partial x}=-K_{e} \frac{\partial p}{\partial t},
$$

where $p, v, \rho$, and $K_{e}$ are the pressure, the particle velocity, the density, and the compressibility of the medium. This study assumes $K_{e}$ to be a constant, i.e., space-and-time independent, though we can conduct a similar study where the density is constant and $K_{e}$ is space-and-time dependent.

The top left panels of Figs. 1(a) and 1(b) illustrate the unit cell for each PC: One has a modulation in the form of $\rho=$ $\rho_{0}\left[1-\alpha \cos \left(k_{m} x\right)\right]$ and the other is $\rho=\rho_{0}\left[1+\alpha \cos \left(k_{m} x\right)\right]$ in the spatial domain $\left[0, \lambda_{m}\right]$, where $\lambda_{m}=2 \pi / k_{m}$ is the space period, $\rho_{0}$ is the air density $\left(1.2 \mathrm{~kg} / \mathrm{m}^{3}\right)$, and $\alpha$ is the modulation amplitude (chosen as $\alpha=0.5$ throughout this study). The two PCs are exactly $180^{\circ}$ out of phase in space. Both PCs have identical band structures (BSs) with a BG around the normalized frequency $\Omega=0.5$ between the first and second bands [bottom left panels of Figs. 1(a) and 1(b)]. For each PC, we plot in the right panels of Figs. 1(a) and 1(b) the acoustic pressure distributions along the unit cell at the edge of the Brillouin zone (BZ) in the first band (point 1), and at both the edge and center of the $\mathrm{BZ}$ in the second band (points 2 and $2^{\prime}$ ). The calculations are performed using the commercial software COMSOL MULTIPHYSICS v5.3a. The symmetry of the eigenmode dictates the Zak phase of each band. For instance, for the first PC in Fig. 1(a), points 2 and $2^{\prime}$ in the second band have the same symmetry, indicating a zero Zak phase; for the second PC in Fig. 2(b), however, different symmetries (antisymmetrical versus symmetrical) are present, implying that the Zak phase is $\pi$. The Zak phase can be also confirmed 


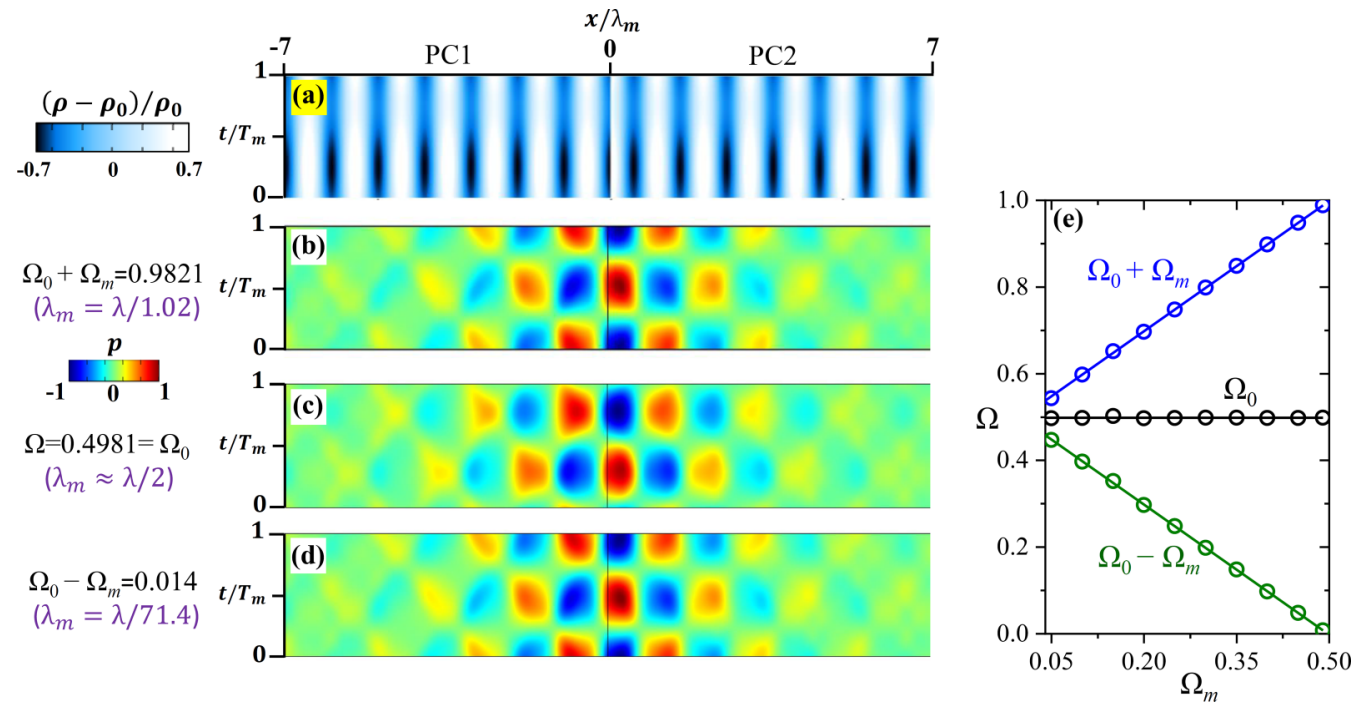

FIG. 2. (a) Density modulation of $\rho=\rho_{0}\left\{1-\left[\alpha+\beta \sin \left(\omega_{m} t\right)\right] \cos \left(k_{m} x\right)\right\}$ for $x<0$ and $\rho=\rho_{0}\left\{1+\left[\alpha+\beta \sin \left(\omega_{m} t\right)\right] \cos \left(k_{m} x\right)\right\}$ for $x>0$ presented within one modulation period $T_{m}$. The wave fields of the edge state (c) inside the BG and duplicated edge states located at (b) $\Omega_{0}+\Omega_{m}$ and (d) $\Omega_{0}-\Omega_{m}$. (e) Frequency of the edge states as function of the modulation frequency $\Omega_{m}$. Lines are theoretical prediction and circles are simulation results.

by using the following formula [24],

$$
\begin{aligned}
\theta_{Z} & =\int_{-\pi / \lambda_{m}}^{\pi / \lambda_{m}}\left\langle u_{k}\left|\partial_{k}\right| u_{k}\right\rangle d k \\
& =\frac{1}{2 \rho_{0} c_{0}^{2}} \int_{-\pi / \lambda_{m}}^{\pi / \lambda_{m}}\left(i \int_{0}^{\lambda_{m}} u_{k}^{*}(x) \partial_{k} u_{k}(x) d x\right) d k,
\end{aligned}
$$

where $k$ is the wave number, $u_{k}=e^{-i k x} p$, and $c_{0}$ is the speed of sound in air $\left(c_{0}=343 \mathrm{~m} / \mathrm{s}\right)$. The pressure eigenfunctions $u_{k}$ are normalized so that $\int_{0}^{\lambda_{m}}\left(1 / \rho_{0} c_{0}^{2}\right)\left|u_{k}\right|^{2} d x=1$ is satisfied.

Taking advantage of the Zak phase change in the second band, a topological edge state located at $x=0$ using both PCs is constructed. The resulting system has a space modulation of $\rho=\rho_{0}\left[1-\alpha \cos \left(k_{m} x\right)\right]$ for $x<0$ and $\rho=$ $\rho_{0}\left[1+\alpha \cos \left(k_{m} x\right)\right]$ for $x>0$. The BS and the transmission spectrum of this system are plotted in Fig. 1(c) which both show the existence of an edge state. This edge state is located at the middle of the Bragg BG, i.e., $\Omega=\omega / k_{m} c_{0}=0.4981$, at which the structure periodicity corresponds to half of the wavelength. We plot in the top panel of Fig. 1(d) the density distribution of the system in a transmission configuration where each PC consists of 7 unit cells (14 in total). For a monochromatic wave excitation at the edge state frequency $\Omega=0.4981$, the pressure field distribution (real part of the complex pressure) is plotted in Fig. 1(d) during one time period $T=2 \pi / \omega$. The acoustic energy is clearly confined in the vicinity of the interface between the two PCs $(x=0)$. It is noted that although sinusoidal space modulation is chosen in this study, the theory developed here can be generalized to other types of modulation (e.g., square modulation) and the main physics is expected to be the same.

\section{SPACE-DECOUPLED TIME MODULATED TOPOLOGICAL PC}

The time modulation is subsequently introduced to both PCs in the following manner:

$$
\rho=\rho_{0}\left\{1-\left[\alpha+\beta \sin \left(\omega_{m} t\right)\right] \cos \left(k_{m} x\right)\right\} \quad \text { for } x<0,
$$

and

$$
\rho=\rho_{0}\left\{1+\left[\alpha+\beta \sin \left(\omega_{m} t\right)\right] \cos \left(k_{m} x\right)\right\} \quad \text { for } x>0,
$$

where $\alpha=0.5$ and $\beta=0.2$ are assumed. A modulation frequency of $\Omega_{m}=\omega_{m} / k_{m} c_{0}=0.484$ is chosen which is close to the edge state's frequency of the stationary system (corresponding to $\beta=0$ ). This enables us to excite an ultralow frequency edge state mode as will be shown later. Please note that the density here is not assumed to be weakly modulated as was done in Ref. [45]. This time modulation is space decoupled in the sense that the functions (sine and cosine) governing the space and time modulations are independent from each other, which is less explored in the past [56]. One can visualize this as the density of the system only having its amplitude varying with time. Figure 2(a) shows the time variation of the reduced density $\left(\rho-\rho_{0}\right) / \rho_{0}$ of the supercell having 14 unit cells. This constitutes the domain in which Eq. (1) is to be solved. With the space-decoupled time modulation of the supercell described by Eq. (3), each PC has its Zak phase fixed with time. This is because only the amplitude of the density is changing with time, so that the symmetry of the second band is conserved at each instant. Consequently, the Zak phase difference between the two PCs remains independent of time. 


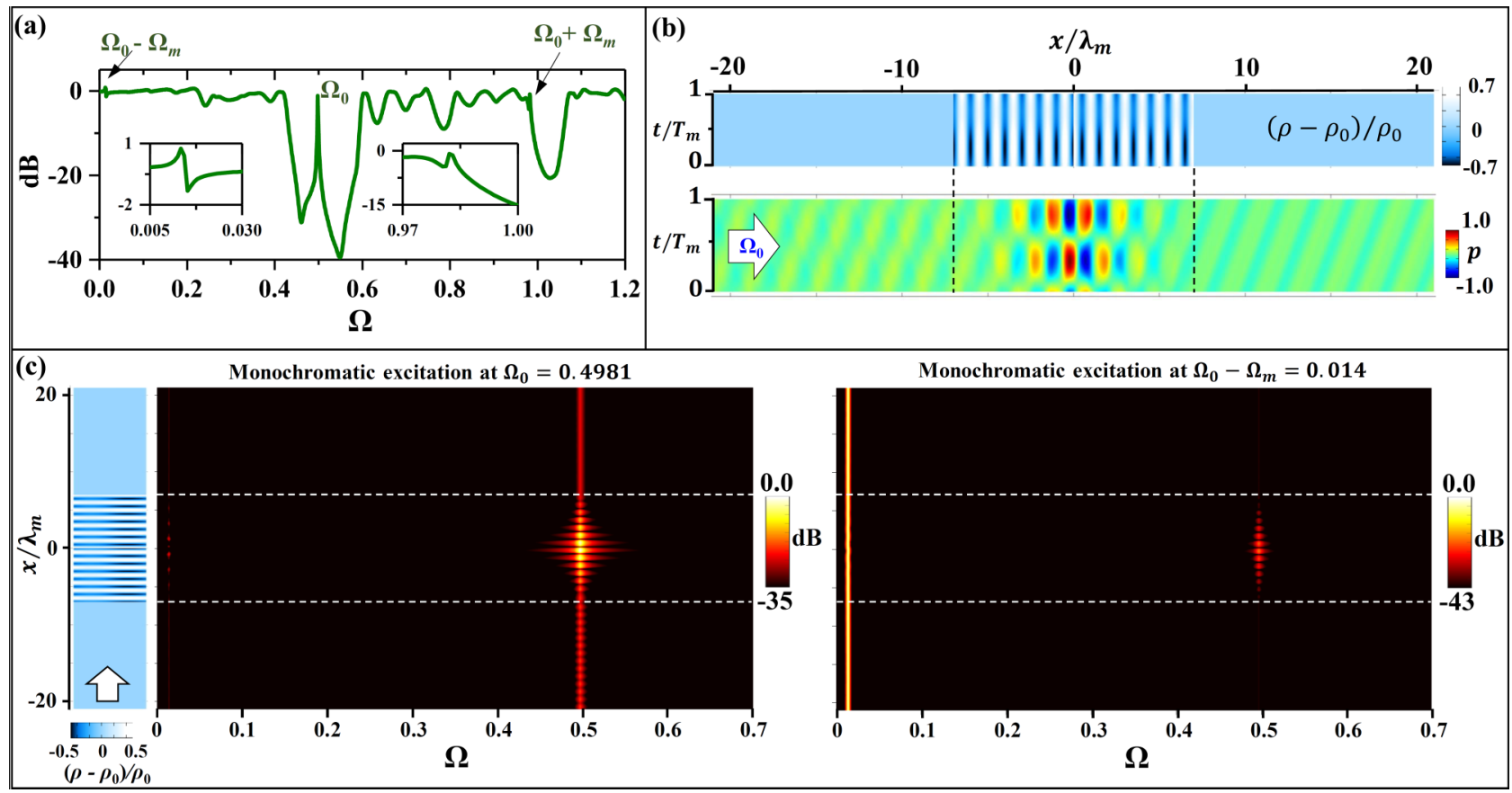

FIG. 3. (a) Transmission spectrum through the space-decoupled time modulated system composed of two PCs with a constant Zak phase shift, and a closeup view near $\Omega_{0} \pm \Omega_{m}$ (inset figures). (b) Top panel: Transmission configuration of the structure with density modulation of $\rho=\rho_{0}\left\{1-\left[\alpha+\beta \sin \left(\omega_{m} t\right)\right] \cos \left(k_{m} x\right)\right\}$ for $x<0$ and $\rho=\rho_{0}\left\{1+\left[\alpha+\beta \sin \left(\omega_{m} t\right)\right] \cos \left(k_{m} x\right)\right\}$ for $x>0$ presented within one modulation period $T_{m}$. Bottom panel: Pressure field for incident wave at $\Omega_{0}=0.4981$. (c) Spatial-frequency spectra of the wave field for incident wave excitations at $\Omega_{0}=0.4981$ (left panel) and $\Omega_{0}-\Omega_{m}=0.014$ (right panel).

Knowing that the density $\rho$ is periodic in both space and time, one can apply the Floquet theorem,

$$
p(x, t)=\tilde{p}(x, t) e^{i(\omega t-k x)}, \quad v(x, t)=\tilde{v}(x, t) e^{i(\omega t-k x)},
$$

where $\tilde{p}$ and $\tilde{v}$ are periodic both in time and space. Inserting these expressions into Eq. (1), the following eigenvalue problem can be constructed where $\omega$ is the eigenvalue to be solved for each wavenumber $k$ :

$$
\begin{aligned}
& \left(\begin{array}{cc}
\frac{\partial}{\partial x} & \rho(x, t) \frac{\partial}{\partial t} \\
K_{e} \frac{\partial}{\partial t} & \frac{\partial}{\partial x}
\end{array}\right)\left(\begin{array}{l}
\tilde{p} \\
\tilde{v}
\end{array}\right)+\left(\begin{array}{cc}
-i k & 0 \\
0 & -i k
\end{array}\right)\left(\begin{array}{l}
\tilde{p} \\
\tilde{v}
\end{array}\right) \\
& =\omega\left(\begin{array}{cc}
0 & -i \rho(x, t) \\
-i K_{e} & 0
\end{array}\right)\left(\begin{array}{l}
\tilde{p} \\
\tilde{v}
\end{array}\right) .
\end{aligned}
$$

The time variable $t$ can be considered as a synthetic dimension in addition to the real physical dimension $x$. Subsequently, Eq. (5) can be solved in the space-time domain displayed in Fig. 2(a) along with the Floquet boundary conditions. Such a method can be generalized to solving wave equation problems involving media with arbitrary space-time periodic properties.

In this study, Eq. (5) is solved again by COMSOL MULTIPHYSICS. Among all possible solutions, we have identified three eigenmodes displayed in Figs. 2(b)-2(d) where the acoustic energy is confined within the vicinity of $x=0$ : The first one is located at frequency $\Omega_{0}=0.4981$ [Fig. 2(c)] and it corresponds to the edge state inside the BG in the stationary case; the second and third modes are located exactly at $\Omega_{0}+$ $\Omega_{m}=0.9821$ [Fig. 2(b)] and $\Omega_{0}-\Omega_{m}=0.014$ [Fig. 2(d)]. These modes are anomalous edge states, and their emergence in the bulk band can be attributed to the time-modulationinduced frequency conversion, which changes the topological feature of the structure leading to duplicated edge states at frequencies $\Omega_{0} \pm n \Omega_{m}$ where $n$ is an integer [52-54]. The positions of these modes in the band can be tuned by changing the modulation frequency $\Omega_{m}$ as shown in Fig. 2(e), where the theory (lines) agrees very well with the simulation (circles).

To gain a better insight on these modes, Fig. 3(a) illustrates the calculated transmission spectrum through the STM system. The calculation is performed in the time domain where we analyze the Fourier spectrum of the transmitted wave through the structure using a wideband incident pulse (a Gaussian-modulated cosine function). A transmission peak corresponding to the edge state is observed inside this BG at the normalized frequency $\Omega_{0}=0.4981$ which corresponds exactly to the stationary case. Note that the system remains reciprocal and the BG does not experience a frequency shift from the stationary case, which is a major departure from the commonly studied space-coupled time modulation where reciprocity is broken [45-47]. In these systems, the space-time perturbation or modulation of the intrinsic property of the medium (density, compressibility, or stiffness) has a driven or wavelike directional motion which breaks the reciprocity. This is not the case for our space-decoupled time modulation which preserves reciprocity.

We also observe two less visible transmission peaks at 0.014 and 0.9821 [insets of Fig. 3(a)] corresponding to the excitation of the first-order edge states outside the BG at $\Omega_{0} \pm \Omega_{m}$, which confirms the prediction of the earlier eigenvalue analysis. These peaks appear less pronounced in the 


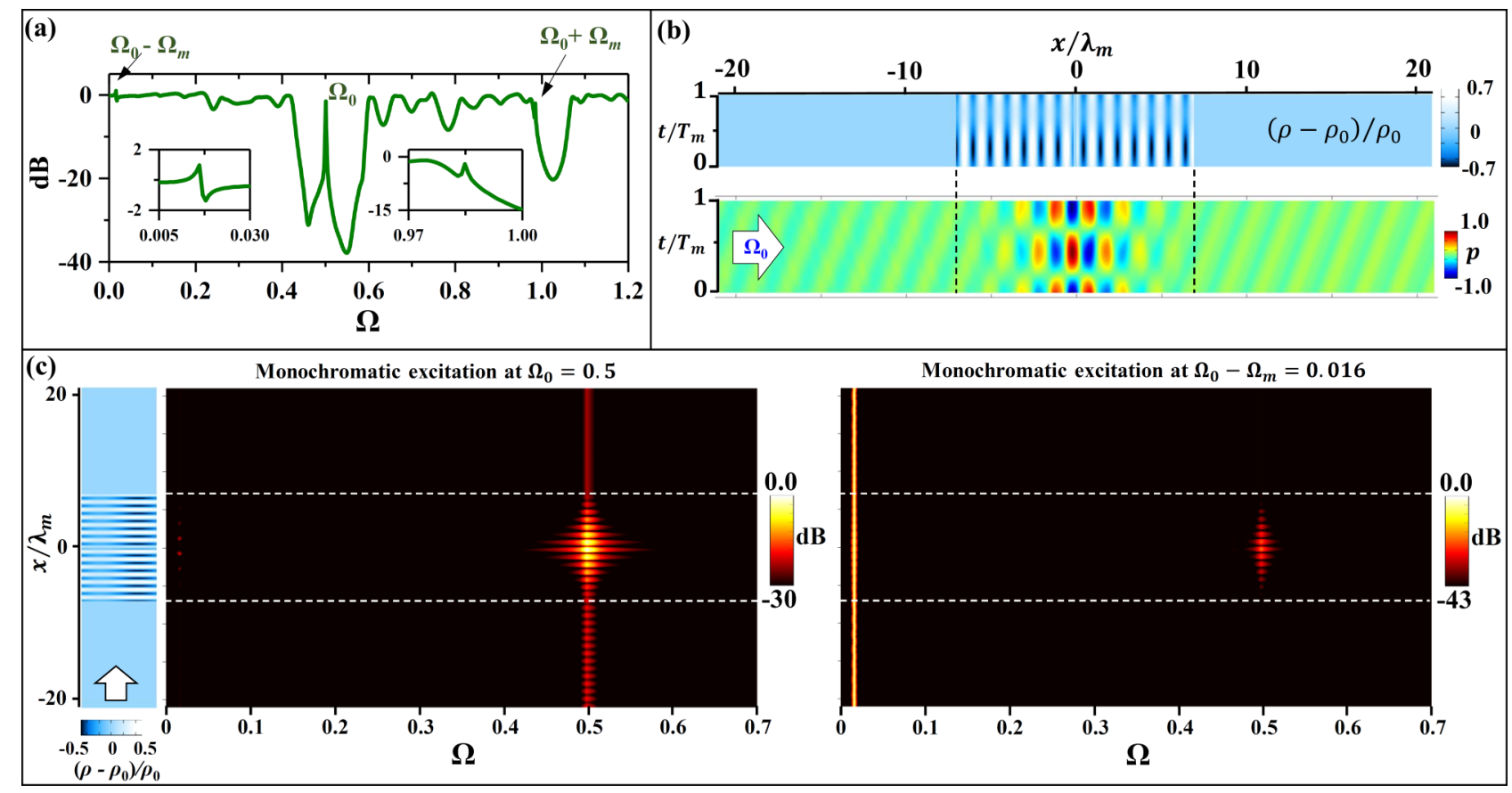

FIG. 4. (a) Transmission spectrum through the space-decoupled time modulated system composed of two PCs and a static density defect in between, and a closeup view near $\Omega_{0} \pm \Omega_{m}$ (inset figures). (b) Top panel: Transmission configuration of the structure with density modulation of $\rho=\rho_{0}\left\{1-\left[\alpha+\beta \sin \left(\omega_{m} t\right)\right] \cos \left(k_{m} x\right)\right\}$ for $x<-\lambda_{m} / 10, \rho=\rho_{0}$ for $-\lambda_{m} / 10<x<\lambda_{m} / 10$, and $\rho=\rho_{0}\left\{1+\left[\alpha+\beta \sin \left(\omega_{m} t\right)\right] \cos \left(k_{m} x\right)\right\}$ for $x>\lambda_{m} / 10$, presented within one modulation period $T_{m}$. Bottom panel: Pressure field for incident wave at $\Omega_{0}=0.5$. (c) Spatial-frequency spectra of the wave field for incident wave excitations at $\Omega_{0}=0.5$ (left panel) and $\Omega_{0}-\Omega_{m}=0.016$ (right panel).

spectrum compared to the one at $\Omega_{0}$ because they are located outside the BG. The bottom panel of Fig. 3(b) shows the wave pressure field for a monochromatic excitation at the frequency $\Omega_{0}=0.4981$ where one can observe the localized edge state mode at the interface $x=0$. This mode, however, is not pure in its Fourier components, although the excitation is strictly monochromatic. To this end, we apply the temporal Fourier transform to the wave field shown in Fig. 3(b) and the results (referred to as the spatial-frequency spectrum of the wave field) are presented in Fig. 3(c) for the case of monochromatic excitations at $\Omega_{0}=0.4981$ and $\Omega_{0}-\Omega_{m}=$ 0.014 , respectively plotted in the left and right panels. In other words, these are the pressure fields along the PC across the frequency spectrum. The spatial domain has a range of $-20<$ $x / \lambda_{m}<20$. In the case of an incident wave at $\Omega_{0}=0.4981$ [left panel of Fig. 3(c)], edge state modes confined at the center of the system (in the vicinity of $x=0$ ) are excited both at this same frequency and at $\Omega_{0}-\Omega_{m}=0.014$, albeit with the latter having a much smaller amplitude. Meanwhile, if we choose a monochromatic excitation exactly at $\Omega_{0}-\Omega_{m}=$ 0.014 , the wave field plotted in the right panel of Fig. 3(c) suggests that it is possible to excite the edge state at $\Omega_{0}=$ 0.4981 inside the BG. We also would like to point out that it is likely that the edge state at $\Omega_{0}-\Omega_{m}$ is also excited but its characteristics (e.g., energy confinement) is not clearly visible as it is "drowned" by the wave field excitation at this frequency. This has happened partially due to the fact that this frequency, again, is located outside the BG.

For the case of monochromatic excitation at $\Omega_{0}-\Omega_{m}=$ 0.014 , the incident wavelength is at $\lambda \approx 71.4 \lambda_{m}$ and the frequency conversion allows the excitation of the edge state inside the BG at $\Omega_{0}=0.4981$ in the Bragg regime. Giving that the entire phononic crystal has a length of $L_{s}=14 \lambda_{m}$, the functionality of the structure is effective though its size is only $1 / 5.1$ of the incident wave wavelength (versus $7 \times$ wavelength in the stationary case). Though the peak pressure of the first-order edge state is about $20 \mathrm{~dB}$ lower than that of the zeroth-order edge state, it is still well within the detectable range under a reasonable signal-to-noise ratio.

Furthermore, an intriguing feature of these edge states is that they are robust against defects both in time and space. In fact, we examine the robustness of the edge states in the space-decoupled time modulated system by considering a defect of size $\lambda_{m} / 5$ in the vicinity of the interface located at $x=0$ between the two PCs, where the density is static. Thus, the whole system has its density following the form

$$
\begin{gathered}
\rho=\rho_{0}\left\{1-\left[\alpha+\beta \sin \left(\omega_{m} t\right)\right] \cos \left(k_{m} x\right)\right\} \text { for } x<-\lambda_{m} / 10, \\
\rho=\rho_{0} \text { for }-\lambda_{m} / 10<x<\lambda_{m} / 10
\end{gathered}
$$

and

$$
\rho=\rho_{0}\left\{1+\left[\alpha+\beta \sin \left(\omega_{m} t\right)\right] \cos \left(k_{m} x\right)\right\} \text { for } x>\lambda_{m} / 10,
$$

where $\alpha=0.5$ and $\beta=0.2$.

We conduct a similar study to show that this defect does not affect the characteristic of the PC. Figure 4(b) shows the density variation during one modulation period $T_{m}=$ $2 \pi / \omega_{m}$ where the defect can be observed as the constant density near $x=0$. Figure 4(a) presents the transmission spectrum for wave propagation through the structure where 
we can clearly see that the curve is very similar to the one of the system without the defect in Fig. 3(a). In fact, the two curves would virtually overlap if we were to plot them together. We can also observe the edge state inside this BG (the peak) at the normalized frequency $\Omega_{0}=0.5$ along with two transmission peaks located at 0.016 and 0.9841 which correspond to the excitation of the first-order edge states outside the BG at $\Omega_{0} \pm \Omega_{m}$. Because of the defect, these frequencies are slightly offset from the original ones $(0.014$, 0.4981 , and 0.9821 ) by a small amount that is roughly 0.002 . We plot in Fig. 4(c) the spatial-frequency spectrum of the wave field for monochromatic excitations at $\Omega_{0}=0.5$ and $\Omega_{0}-\Omega_{m}=0.016$, respectively, in the left and right panels. We can observe the same results as in Fig. 3(c) for the system without the defect. The edge states, including the anomalous ones, are therefore topologically protected against the defect and can be excited at a deep subwavelength scale where the incident wavelength is $\lambda \approx 62.5 \lambda_{m}$ in this case.

\section{SPACE-COUPLED TIME MODULATED TOPOLOGICAL PC}

We investigate here the wave propagation for the space-coupled time modulated system. In this case, the time modulation is introduced to both PCs following $\rho=\rho_{0}\left[1-\alpha \cos \left(\omega_{m} t-k_{m} x\right)\right]$ for $x<0$ and $\rho=$ $\rho_{0}\left[1+\alpha \cos \left(\omega_{m} t-k_{m} x\right)\right]$ for $x>0$. A modulation frequency of $\Omega_{m}=\omega_{m} / k_{m} c_{0}=0.2$ is chosen. We choose this number so that we can also demonstrate high-order edge state modes (high order in terms of the frequency). In the previous case, we have only demonstrated the zeroth- and first-order edge state modes. This time modulation is space coupled in the sense that both ST modulations appear in the cosine function. Contrary to the space-decoupled time system, one major difference is that the space-coupled modulation breaks the reciprocity. This is because under such a modulation, the density variation can be manifested as a wave propagating in either positive or negative $x$ direction [see Fig. 6(b), top panel], and this synthetic external field is the foundation of the mechanism that breaks the symmetry of the system.

With the space-coupled time modulation, each PC has its Zak phase changing with time in a way that the Zak phase difference remains constant between the two PCs. Figure 5 displays the Zak phase variation against time for each PC during one modulation period $T_{m}=2 \pi / \omega_{m}$, where we can clearly see that the Zak phase difference between the two PCs remains constant at $\pi$, indicating the existence of edge states at each time instant.

The top panel of Fig. 6(b) shows the density variation during one modulation period $T_{m}=2 \pi / \omega_{m}$ where the topological system is made of 14 unit cells, the same as in the stationary case (Fig. 1). Indeed, the density variation can be seen as a wave traveling in the $+x$ direction as time progresses. Figure 6(a) illustrates the calculated transmission spectrum for forward wave propagation (from left to right). Compared to the stationary case and the space-decoupled time modulated system, the BG under the space-coupled time modulation is shifted to higher frequencies by approximately $\Omega_{m} / 2=0.1$. It was previously shown that this type of time

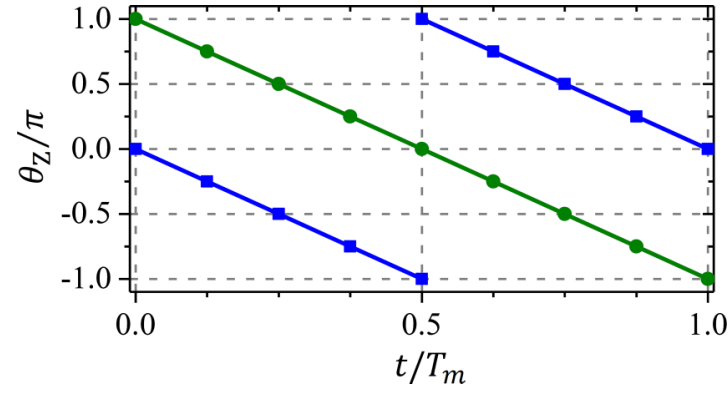

FIG. 5. Zak phase $\theta_{z}$ as a function of time for both PCs during one modulation period. The blue line is for the first PC with the modulation of $\rho=\rho_{0}\left[1+\alpha \cos \left(\omega_{m} t-k_{m} x\right)\right]$ while the green line is for the second PC with the modulation of $\rho=\rho_{0}[1-$ $\left.\alpha \cos \left(\omega_{m} t-k_{m} x\right)\right]$. The difference between the two Zak phases remains unchanged and is equal to $\pi$.

modulation could introduce an asymmetrical BG shift for opposite propagation directions which can be used to achieve one-way propagation (nonreciprocity) [45-47]. Furthermore, in Fig. 6(a), a transmission peak is observed inside this BG at the normalized frequency $\Omega=0.6013$, corresponding to the excitation of the edge state which is also shifted from its initial frequency in the case of the stationary system $(\Omega=0.4981)$. We also note two transmission dips at $\Omega=0.2013$ and 0.4013 as well as a peak at 0.8013 and all of them are outside the BG. which correspond to the excitation of high-order edge states.

In the same way as in the space-decoupled time modulated system, we perform transmission calculations in the time domain where we analyze the Fourier spectrum of the transmitted wave through the structure. The bottom panels of Fig. 6(b) show the wave pressure fields for monochromatic excitations at frequencies $\Omega_{0}=0.6013$ and $\Omega_{0}-\Omega_{m}=0.4013$, where one can observe localized modes at the interface. To these wave fields, we apply the temporal Fourier transform and the results are presented in Fig. 6(c) for the case of $\Omega_{0}=$ $0.6013, \Omega_{0}-\Omega_{m}=0.4013$, and also $\Omega_{0}-2 \Omega_{m}=0.2013$, respectively plotted in the top left, top right, and bottom panels. In the case of an incident wave at $\Omega_{0}=0.6013$ [top left panel of Fig. 6(c)], an edge state mode confined at the center of the system (in the vicinity of $x=0$ ) is excited at $\Omega_{0}=0.6013$ but also at $\Omega_{0}-\Omega_{m}=0.4013$ which is outside the BG. A second-order edge state is also excited at $\Omega_{0}$ $2 \Omega_{m}=0.2013$ but is far less visible since the energy allocated at higher-order edge states is weaker. Meanwhile, if we choose a monochromatic excitation exactly at $\Omega_{0}-\Omega_{m}=0.4013$, the wave fields plotted in the top right panel of Fig. 6(c) suggest that, not only the excitation of an edge state at this frequency $(\Omega=0.4013$ ) is possible, but also an edge state at $\Omega_{0}=0.6013$ inside the BG can be excited. This behavior is similar to the one observed for the space-decoupled time modulated system. In the same way, if the excitation is at $\Omega_{0}-2 \Omega_{m}=0.2013$ [bottom panel of Fig. 6(c)], it is possible to excite the edge states at $\Omega_{0}-\Omega_{m}=0.4013$ and $\Omega_{0}=$ 0.6013 simultaneously. In this particular case, the edge state inside the Bragg BG is excited using a monochromatic wave at a wavelength $\lambda \approx 4.97 \lambda_{m}$. 


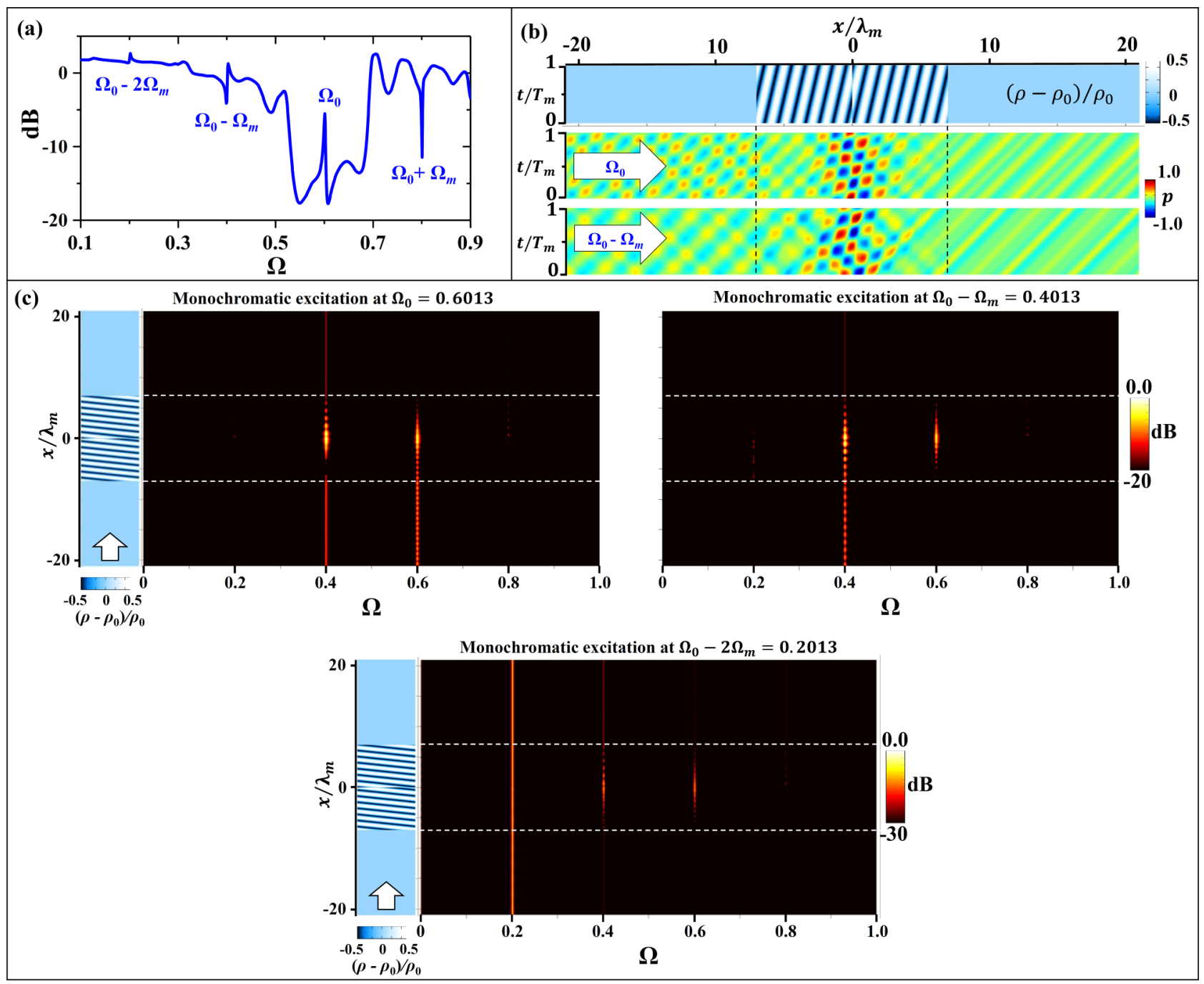

FIG. 6. (a) Transmission spectrum through the space-coupled time modulated system composed of two PCs with a constant Zak phase shift. (b) Top panel: Transmission configuration of the structure with density modulation of $\rho=\rho_{0}\left[1-\alpha \cos \left(\omega_{m} t-k_{m} x\right)\right]$ for $x<0$ and $\rho=\rho_{0}\left[1+\alpha \cos \left(\omega_{m} t-k_{m} x\right)\right]$ for $x>0$ presented within one modulation period $T_{m}$. Bottom panels: Pressure fields for incident wave at $\Omega_{0}=0.6013$ and $\Omega_{0}-\Omega_{m}=0.4013$, respectively. (c) Spatial-frequency spectra of the wave field for incident wave excitations at $\Omega_{0}=0.6013$ (top left panel), $\Omega_{0}-\Omega_{m}=0.4013$ (top right panel), and $\Omega_{0}-2 \Omega_{m}=0.2013$ (bottom panel).

Meanwhile, the transmission characteristic of the system for backward wave propagation is dramatically different due to the nonreciprocity of the system. Figures 7 (a) and 7 (b) illustrate the calculated transmission spectra for forward (from left to right) and backward (from right to left) wave propagation, respectively. Compared to the stationary case presented in Fig. 1, the BG under the space-coupled time modulation is shifted to higher frequencies by $\Omega_{m} / 2=0.1$ in the case of forward propagation [Fig. 7(a)], while it is shifted to lower frequencies by $-\Omega_{m} / 2=-0.1$ for backward propagation [Fig. 7(b)].

The system's nonreciprocity for wave propagation can lead to multiple functionalities. For instance, the structure can be utilized for or unidirectional sensing using either the peak at $\Omega_{0}=0.6$ inside the BG which extends from $\Omega=0.52$ to 0.69 for forward propagation [Fig. 7(a)] or in the backward propagation using the peak at $\Omega=0.4$ [Fig. 7(b)] inside the $\mathrm{BG}$ which ranges from 0.32 to 0.49 . Additionally, the backward propagation can be used for unidirectional filtering using the second $\mathrm{BG}$ which ranges from $\Omega=0.77$ to 0.85 [Fig. 7(b)] while in this same frequency range the structure behaves as a single frequency selective filter for forward propagation at $\Omega_{0}+\Omega_{m}=0.8$ [Fig. 7(a)]. It is noted that all these functionalities can be tuned by changing the modulation frequency.

\section{CONCLUSION}

In conclusion, we propose to enrich the functionalities of topological devices via time modulation, which gives rise to the duplication of topological edge states across the entire spectrum. This feature can be used to achieve deep subwavelength manipulation of the edge state in the Bragg regime, which could be proven useful for designing miniature topological devices for waveguiding, sensing, and excitation, among other intriguing functionalities. Furthermore, we show that the time modulation can be introduced to break reciprocity that, in 


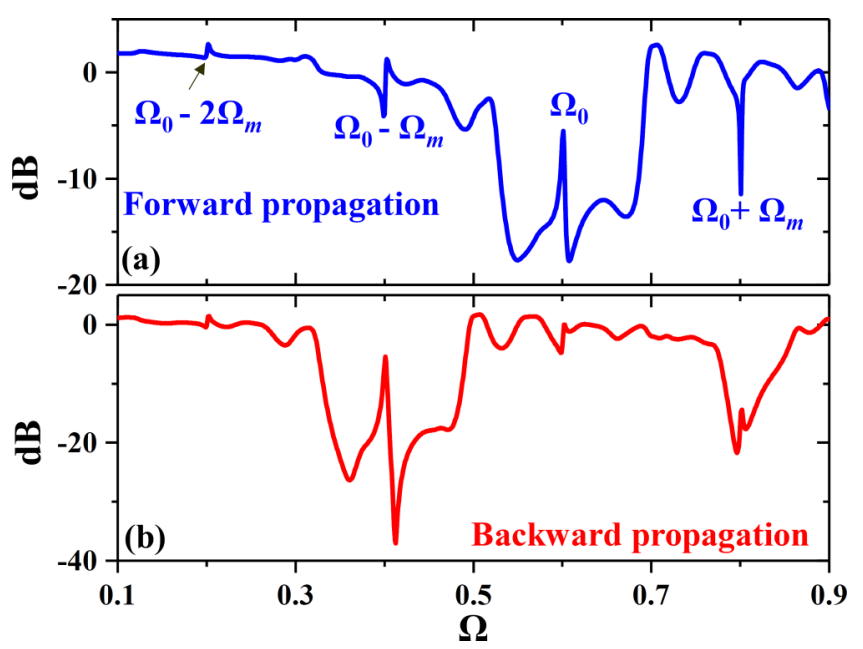

FIG. 7. (a) Transmission spectrum through the space-coupled time modulated system composed of two PCs with a constant Zak phase shift for (a) forward and (b) backward propagation. conjunction with the anomalous edge states, could engender exotic unidirectional wave behaviors. Future work will expand the model to higher dimensions where even richer physics can be foreseen. It would be also interesting to construct a real physical model to experimentally validate the theory. Active acoustic metamaterials [45] and piezoelectric materials $[49,50]$ could be viable options in this regard. For instance, one can imagine a one-dimensional acoustic metamaterial similar to the one introduced by Chen et al. [57]. Using the periodic distribution of membranes connected to electromagnets that change the membrane tension, it is possible to tune the effective acoustic density by almost $100 \%$ upon changing the applied voltage.

\section{ACKNOWLEDGMENTS}

The authors would like to thank Dr. Meng Xiao for his critical comments on the paper. M.T. acknowledges the generous support from NSF DMS-1521667, DMS-1847802, and ECCS-1936776.
[1] F. D. M. Haldane and S. Raghu, Possible Realization of Directional Optical Waveguides in Photonic Crystals with Broken Time-Reversal Symmetry, Phys. Rev. Lett. 100, 013904 (2008).

[2] M. Hafezi, E. A. Demler, M. D. Lukin, and J. M. Taylor, Robust optical delay lines with topological protection, Nat. Phys. 7, 907 (2011).

[3] K. Fang, Z. Yu, and S. Fan, Realizing effective magnetic field for photons by controlling the phase of dynamic modulation, Nat. Photonics 6, 782 (2012).

[4] L.-H. Wu and X. Hu, Scheme for Achieving a Topological Photonic Crystal by Using Dielectric Material, Phys. Rev. Lett. 114, 223901 (2015).

[5] A. B. Khanikaev, S. H. Mousavi, W.-K. Tse, M. Kargarian, A. H. MacDonald, and G. Shvets, Photonic topological insulators, Nat. Mater. 12, 233 (2013).

[6] M. C. Rechtsman, J. M. Zeuner, Y. Plotnik, Y. Lumer, D. Podolsky, F. Dreisow, S. Nolte, M. Segev, and A. Szameit, Photonic Floquet topological insulators, Nature (London) 496, 196 (2013)

[7] L. Lu, J. D. Joannopoulos, and M. Soljačić, Topological photonics, Nat. Photonics 8, 821 (2014).

[8] Y. Plotnik, M. C. Rechtsman, D. H. Song, M. Heinrich, J. M. Zeuner, S. Nolte, Y. Lumer, N. Malkova, J. Xu, A. Szameit, Z. Chen, and M. Segev, Observation of unconventional edge states in "photonic graphene", Nat. Mater. 13, 57 (2014).

[9] S. A. Skirlo, L. Lu, and M. Soljačić, Multimode One-Way Waveguides of Large Chern Numbers, Phys. Rev. Lett. 113, 113904 (2014).

[10] S. A. Skirlo, L. Lu, Y. Igarashi, Q. Yan, J. Joannopoulos, and M. Soljačić, Experimental Observation of Large Chern Numbers in Photonic Crystals, Phys. Rev. Lett. 115, 253901 (2015).

[11] T. Ozawa, H. M. Price, A. Amo, N. Goldman, M. Hafezi, L. Lu, M. C. Rechtsman, D. Schuster, J. Simon, O. Zilberberg, and I. Carusotto, Topological photonics, Rev. Mod. Phys. 91, 015006 (2019).
[12] Z. Yang, F. Gao, X. Shi, X. Lin, Z. Gao, Y. Chong, and B. Zhang, Topological Acoustics, Phys. Rev. Lett. 114, 114301 (2015).

[13] X. Ni, C. He, X.-C. Sun, X.-P Liu, M.-H. Lu, L. Feng, and Y.-F. Chen, Topologically protected one-way edge mode in networks of acoustic resonators with circulating air flow, New J. Phys. 17, 053016 (2015).

[14] A. B. Khanikaev, R. Fleury, S. H. Mousavi, and A. Alù, Topologically robust sound propagation in an angular-momentumbiased graphene-like resonator lattice, Nat. Commun. 6, 8260 (2015).

[15] C. He, X. Ni, H. Ge, X.-C. Sun, Y.-B. Chen, M.-H. Lu, X.-P. Liu, and Y.-F. Chen, Acoustic topological insulator and robust one-way sound transport, Nat. Phys. 12, 1124 (2016).

[16] R. Fleury, A. B. Khanikaev, and A. Alù, Floquet topological insulators for sound, Nat. Commun. 7, 11744 (2016).

[17] Z.-G. Chen and Y. Wu, Tunable Topological Phononic Crystals, Phys. Rev. Applied 5, 054021 (2016).

[18] Y. Deng, H. Ge, Y. Tian, M. Lu, and Y. Jing, Observation of zone folding induced acoustic topological insulators and the role of spin-mixing defects, Phys. Rev. B 96, 184305 (2017).

[19] X. Zhang, M. Xiao, Y. Cheng, M.-H. Lu, and J. Christensen, Topological sound, Commun. Phys. 1, 97 (2018).

[20] G. Ma, M. Xiao, and C. T. Chan, Topological phases in acoustic and mechanical systems, Nat. Rev. Phys. 1, 281 (2019).

[21] H. Ge, X. Ni, Y. Tian, S. K. Gupta, M.-H. Lu, X. Lin, W.-D. Huang, C. T. Chan, and Y.-F. Chen, Experimental Observation of Acoustic Weyl Points and Topological Surface States, Phys. Rev. Applied 10, 014017 (2018).

[22] C. He, S.-Y. Yu, H. Ge, H. Wang, Y. Tian, H. Zhang, X.-C. Sun, Y. B. Chen, J. Zhou, M.-H. Lu, and Y.-F. Chen, Threedimensional topological acoustic crystals with pseudospinvalley coupled saddle surface states, Nat. Commun. 9, 4555 (2018).

[23] F. Li, X. Huang, J. Lu, J. Ma, and Z. Liu, Weyl points and Fermi arcs in a chiral phononic crystal, Nat. Phys. 14, 30 (2018). 
[24] M. Xiao, G. Ma, Z. Yang, P. Sheng, Z. Q. Zhang, and C. T. Chan, Geometric phase and band inversion in periodic acoustic systems, Nat. Phys. 11, 240 (2015).

[25] X. Li, Y. Meng, X. Wu, S. Yan, Y. Huang, S. Wang, and W. Wen, Su-Schrieffer-Heeger model inspired acoustic interface states and edge states, Appl. Phys. Lett. 113, 203501 (2018).

[26] W. Zhu, X. Fang, D. Li, Y. Sun, Y. Li, Y. Jing, and H. Chen, Simultaneous Observation of a Topological Edge State and Exceptional Point in an Open and Non-Hermitian Acoustic System, Phys. Rev. Lett. 121, 124501 (2018).

[27] X. Ni, M. Weiner, A. Alù, and A. B. Khanikaev, Observation of higher-order topological acoustic states protected by generalized chiral symmetry, Nat. Mater. 18, 113 (2019).

[28] H. Xue, Y. Yang, F. Gao, Y. Chong, and B. Zhang, Acoustic higher-order topological insulator on a kagome lattice, Nat. Mater. 18, 108 (2019).

[29] S. H. Mousavi, A. B. Khanikaev, and Z. Wang, Topologically protected elastic waves in phononic metamaterials, Nat. Commun. 6, 8682 (2015).

[30] S.-Y. Yu, C. He, Z. Wang, F.-K. Liu, X.-C. Sun, Z. Li, H.Z. Lu, M.-H. Lu, X.-P. Liu, and Y.-F. Chen, Elastic pseudospin transport for integratable topological phononic circuits, Nat. Commun. 9, 3072 (2018).

[31] M. Miniaci, R. K. Pal, B. Morvan, and M. Ruzzene, Experimental Observation of Topologically Protected Helical Edge Modes in Patterned Elastic Plates, Phys. Rev. X 8, 031074 (2018).

[32] M. Yan, J. Lu, F. Li, W. Deng, X. Huang, J. Ma, and Z. Liu, Onchip valley topological materials for elastic wave manipulation, Nat. Mater. 17, 993 (2018).

[33] Z. Wang, F.-K. Liu, S.-Y. Yu, S.-L. Yan, M.-H. Lu, Y. Jing, and Y.-F. Chen, Guiding robust valley-dependent edge states by surface acoustic waves, J. Appl. Phys. 125, 044502 (2019).

[34] J. Zak, Berry's Phase for Energy Bands in Solids, Phys. Rev. Lett. 62, 2747 (1989).

[35] M. Atala, M. Aidelsburger, J. T. Barreiro, D. Abanin, T. Kitagawa, E. Demler, and I. Bloch, Direct measurement of the Zak phase in topological Bloch bands, Nat. Phys. 9, 795 (2013).

[36] A. Oliner and A. Hessel, Guided waves on sinusoidally modulated reactance surfaces, IEEE Trans Antennas Propag. 7, 201 (1959).

[37] E. S. Cassedy and A. A. Oliner, Dispersion relations in timespace periodic media: Part I-Stable interactions, Proc. IEEE 51, 1342 (1963).

[38] E. S. Cassedy, Waves guided by a boundary with time-space periodic modulation, Proc. IEE 112, 269 (1965).

[39] Y. Hadad, D. L. Sounas, and A. Alù, Space-time gradient metasurfaces, Phys. Rev. B 92, 100304(R) (2015).

[40] S. Taravati, Self-biased broadband magnet-free linear isolator based on one-way space-time coherency, Phys. Rev. B 96, 235150 (2017).

[41] S. Taravati, N. Chamanara, and C. Caloz, Nonreciprocal electromagnetic scattering from a periodically space-time modulated slab and application to a quasisonic isolator, Phys. Rev. B 96, 165144 (2017).

[42] S. Taravati, Aperiodic space-time modulation for pure frequency mixing, Phys. Rev. B 97, 115131 (2018).

[43] S. Taravati, Giant Linear Nonreciprocity, Zero Reflection, and Zero Band Gap in Equilibrated Space-Time-Varying Media, Phys. Rev. Applied 9, 064012 (2018).

[44] R. Fleury, D. L. Sounas, and A. Alù, Subwavelength ultrasonic circulator based on spatiotemporal modulation, Phys. Rev. B 91, 174306 (2015).

[45] C. Shen, J. Li, Z. Jia, Y. Xie, and S. A. Cummer, Nonreciprocal acoustic transmission in cascaded resonators via spatiotemporal modulation, Phys. Rev. B 99, 134306 (2019).

[46] J. Li, C. Shen, X. Zhu, Y. Xie, and S. A. Cummer, Nonreciprocal sound propagation in space-time modulated media, Phys. Rev. B 99, 144311 (2019).

[47] G. Trainiti and M. Ruzzene, Non-reciprocal elastic wave propagation in spatiotemporal periodic structures, New J. Phys. 18, 083047 (2016).

[48] Y. Wang, B. Yousefzadeh, H. Chen, H. Nassar, G. Huang, and C. Daraio, Observation of Nonreciprocal Wave Propagation in a Dynamic Phononic Lattice, Phys. Rev. Lett. 121, 194301 (2018).

[49] G. Trainiti, Y. Xia, J. Marconi, G. Cazzulani, A. Erturk, and M. Ruzzene, Time-Periodic Stiffness Modulation in Elastic Metamaterials for Selective Wave Filtering: Theory and Experiment, Phys. Rev. Lett. 122, 124301 (2019).

[50] K. Yi, M. Ouisse, E. Sadoulet-Reboul, and G. Matten, Active metamaterials with broadband controllable stiffness for tunable band gaps and non-reciprocal wave propagation, Smart Mater. Struct. 28, 065025 (2019).

[51] N. H. Lindner, G. Refael, and V. Galitski, Floquet topological insulator in semiconductor quantum wells, Nat. Phys. 7, 490 (2011).

[52] T. Kitagawa, E. Berg, M. Rudner, and E. Demler, Topological characterization of periodically driven quantum systems, Phys. Rev. B 82, 235114 (2010).

[53] M. S. Rudner, N. H. Lindner, E. Berg, and M. Levin, Anomalous Edge States and the Bulk-Edge Correspondence for Periodically Driven Two-Dimensional Systems, Phys. Rev. X 3, 031005 (2013).

[54] Z. Fedorova, C. Jörg, C. Dauer, F. Letscher, M. Fleischhauer, S. Eggert, S. Linden, and G. von Freymann, Limits of topological protection under local periodic driving, Light: Sci. Appl. 8, 63 (2019).

[55] P. M. Morse and K. U. Ingard, Theoretical Acoustics (McGraw-Hill, New York, 1968).

[56] E. Riva, J. Marconi, G. Cazzulani, and F. Braghin, Generalized plane wave expansion method for non-reciprocal discretely modulated waveguides, J. Sound Vib. 449, 172 (2019).

[57] Z. Chen, C. Xue, L. Fan, S.-Y. Zhang, X.-J. Li, H. Zhang, and J. Ding, A tunable acoustic metamaterial with double-negativity driven by electromagnets, Sci. Rep. 6, 30254 (2016). 